\title{
A New Technology for Fast Two-Dimensional Detection of Proton Therapy Beams
}

\author{
Robert Hollebeek, ${ }^{1}$ Mitch Newcomer, ${ }^{1}$ Godwin Mayers, ${ }^{1}$ Brian Delgado, ${ }^{1}$ Gaurav Shukla, ${ }^{1}$ \\ Richard Maughan, ${ }^{2}$ and Derek Dolney ${ }^{2}$ \\ ${ }^{1}$ Department of Physics, University of Pennsylvania, Philadelphia, PA 19104, USA \\ ${ }^{2}$ Department of Radiation Oncology, University of Pennsylvania, Philadelphia, PA 19104, USA
}

Correspondence should be addressed to Robert Hollebeek, bobh@physics.upenn.edu

Received 20 July 2012; Accepted 20 November 2012

Academic Editor: Flavio P. Marchetto

Copyright (C) 2012 Robert Hollebeek et al. This is an open access article distributed under the Creative Commons Attribution License, which permits unrestricted use, distribution, and reproduction in any medium, provided the original work is properly cited.

\begin{abstract}
The Micromesh Gaseous Structure, or Micromegas, is a technology developed for high count-rate applications in high-energy physics experiments. Tests using a Micromegas chamber and specially designed amplifiers and readout electronics adapted to the requirements of the proton therapy environment and providing both excellent time and high spatial resolution are presented here. The device was irradiated at the Roberts Proton Therapy Center at the University of Pennsylvania. The system was operated with ionization gains between 10 and 200 and in low and intermediate dose-rate beams, and the digitized signal is found to be reproducible to $0.8 \%$. Spatial resolution is determined to be $1.1 \mathrm{~mm}(1 \sigma)$ with a $1 \mathrm{~ms}$ time resolution. We resolve the range modulator wheel rotational frequency and the thicknesses of its segments and show that this information can be quickly measured owing to the high time resolution of the system. Systems of this type will be extremely useful in future treatment methods involving beams that change rapidly in time and spatial position. The Micromegas design resolves the high dose rate within a proton Bragg peak, and measurements agree with Geant 4 simulations to within $5 \%$.
\end{abstract}

\section{Introduction}

At the time of this writing there are 40 hadron therapy facilities in operation worldwide treating cancer patients with hadrons. Nearly 100,000 patients have completed treatments [1]. The path of a hadron in tissue is essentially straight with relatively low energy loss until very near the end of the range where most energy is deposited in a small region called the Bragg peak, with little exit dose relative to photons. The depth of the Bragg peak in the patient is controlled by the beam energy, and modern delivery systems allow fast and continuous modulation of beam energy during delivery. Collimation of treatment fields achieves good lateral dose gradients, comparable to X-ray fields, and systems of detectors exist to measure 2D planes of dose distributions. The steep longitudinal dose gradient that is the motivation behind proton therapy additionally allows for high dose conformity in a third dimension, but detector systems for characterizing 3D dose distributions do not exist. As an alternative to collimation, narrow proton pencil beams can be scanned magnetically to provide even better dose localization [2]. Although pencil-beam scanning has been used for only a few percent of all hadron treatments to date, a number of the next centers proposed or under construction are planned to offer only the scanned-beam technique. Due to the potential for interplay between the motion of the pencil beam and the respiratory motion of the patient, it is necessary to resolve the time structure of the dose delivery to fully realize the capabilities of hadron therapy, particularly for lung tumors. Direct detection of the high beam current used for hadron therapies requires a new generation of dosimetry devices capable of high spatial and time resolution accompanied by good linearity and little to no saturation in the Bragg peak. New dosimetry technology tailored to hadron therapy will

(i) reduce the uncertainties in beam characteristics (position, energy/range, stability), partially addressing the range uncertainty problem and potentially 
allowing for a reduction in treatment margins, thereby permitting additional disease sites to be treated with particle beams, improving the prospects for dose escalation and hypofractionation strategies, and generally lowering the integral dose to normal tissues across all disease sites,

(ii) accelerate the development and optimization of the next generation of dose delivery technologies, including intensity-modulated scanned pencil beams, especially in conjunction with patient motion management and image-guided radiation therapy (IGRT) systems, by providing good spatial and time resolution for the dose delivery,

(iii) assist in the development of new and more advanced therapy-supporting technologies, and become key components of those technologies including proton radiography [3], proton-computed tomography [46], range verification [7], and range probes [8], where high spatial and time resolution are important requirements,

(iv) facilitate more rapid delivery and commissioning of new particle therapy facilities, and

(v) allow more comprehensive Quality Assurance (QA) measurements of patient treatment plans to be performed $[9,10]$.

This paper describes the design and performance of a new device with millimeter and millisecond resolution and high dynamic range. We will be discussing proton therapy in particular, though our design could be adapted for other particle types. The requirements for an ideal beam monitoring device used for the characterization of proton therapy beams are stringent: beam position should be resolved at the order of about $1 \mathrm{~mm}$ and dose measured with an accuracy of $2 \%$ or better. Ionization chambers represent the gold standard for dose accuracy since the gain depends only on ionization in the gas mixture used. Good spatial resolution however requires multiple chambers. The high amplifier gain required to detect the small signals leads to limited bandwidth and therefore limited time resolution. If using a single chamber, it must be repositioned to obtain beam profiles. This is very time consuming whether done manually or using stepping motors. Multiple devices can be placed in the beamline to obtain some spatial resolution from a single beam delivery, and one and two dimensional arrays of ionization chambers are now available commercially and useful for proton therapy measurements. Semiconductor detectors are also a possible choice for proton therapy dosimetry as they can be made small and thus provide good spatial resolution, and they can be read out fast for good time resolution; however, they tend to be expensive for large volume arrays, suffer degradation from radiation exposure, and have a response depending on energy, temperature, and dose rate. Detector systems based on scintillating materials can be manufactured at relatively low cost and can provide good resolution in 2D using planes of scintillating material [11] and in $3 \mathrm{D}$ using a volume of scintillator [12]. Thus far, however, scintillating devices used in proton therapy beams suffer from radiation damage and some saturation in the Bragg peak, though it appears that corrections can be made to obtain a reasonable calibration. Chemical dosimeters, including ferrous (Fricke) gels and polymer gels, can be poured into containers of different sizes and shapes and irradiated, followed by readout by methods such as MRI or optical CT, but these, like film, are strictly integrative materials that offer no time resolution.

A typical proton therapy irradiation will deliver $2 \mathrm{~Gy}$ to a $1 \mathrm{~L}$ volume in 1-2 minutes, mostly by direct ionization of tissue molecules, meaning that ion pairs are created in the volume at a rate of a few hundred $\mu \mathrm{A}$. A small chamber with active volume $1 \mathrm{~mm}^{3}$ would see only a few hundred $\mathrm{pA}$. The dose profile at the edges of the treatment field falls off like an error function, and ideally one would like to resolve the dose in this penumbra at the level of $1 \%$ of the maximum dose. Therefore, the goal of a dosimeter for proton therapy is to resolve pA-scale currents. In the case of actively scanned beams, the beam may be swept across the chamber volume on millisecond timescales.

Compared to ionization chambers, proportional counters provide larger signals by operating at a sufficiently high field strength such that primary ions in the gas are accelerated enough to produce additional ionization. Counters operating in the proportional region achieve high enough gain for sensitivity to single ionizing particles. Multiwire proportional chambers and drift chambers can be fabricated into devices that cover large volumes. While many traditional chambers of this type were constructed with small diameter wires, there are now competing technologies for this application, including GEM detectors where the small structures producing the high electric fields are holes, and Micromesh Gaseous Structure, or so-called Micromegas, where the structures are fine meshes [13]. The Micromegas is a variation of an ionization chamber where a fine mesh has been introduced to create a high-field region where large gas gains, up to factors of $10^{6}$, can be realized [14-16]. Advantages of this approach include large dynamic range, high rate capability, good time resolution, and fine segmentation. A manufacturing process to produce Micromegas chambers in bulk at relatively low cost has matured [17], and refinements to the basic design continue to develop [18]. In this work, we present a novel adaptation of the Micromegas technology for the proton therapy environment, where the device is operated at low gain and is operated in current mode rather than pulse mode.

In this paper we describe the configuration and testing of a multichannel Micromegas detector that has been designed and assembled at the University of Pennsylvania and irradiated in the proton therapy beam at the Roberts Proton Therapy Center. We present measurements of spatial and time resolution and show that the chamber resolves a proton Bragg peak without saturation. To our knowledge, this is the first data obtained with this type of detector in a proton therapy beam.

\section{Methods and Materials}

A prototype Micromegas chamber with a segmented anode plane was designed and assembled at the University of 


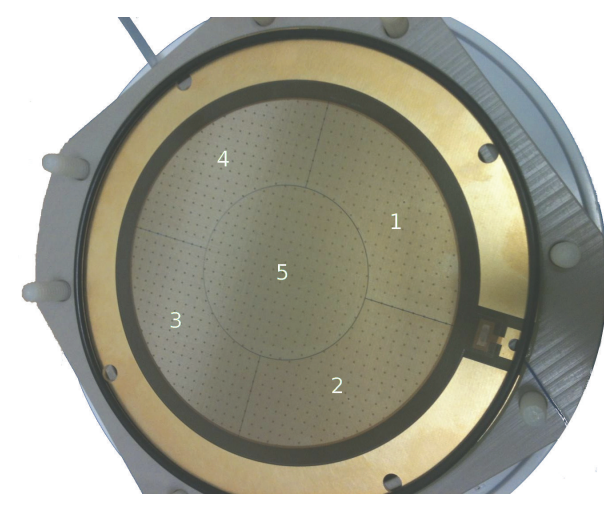

Figure 1: The Micromegas board. The five readout anodes can be seen in the center and are numbered. The woven micromesh is held above the readout anodes by photo-etched Kapton standoffs (visible as small points covering the anode plane) that maintain a uniform $128 \mu \mathrm{m}$ gap. The center channel is $5 \mathrm{~cm}$ in diameter. Also visible is the insulating ring, O-ring for gas seal, high voltage lead (lower right), and gas feed pipe (upper left).

Pennsylvania. The mesh and anode layer were fabricated at CERN following the bulk Micromegas process described elsewhere [17]. The chamber has a circular center channel and four peripheral quadrants (see Figure 1) etched on one side of a standard $2 \mathrm{oz}$ double-sided copper clad G10 printed circuit board (PCB). The segments are electrically connected to readout channels by plate-through holes to the back of the PCB. The micromesh is a woven stainless steel mesh of $20 \mu \mathrm{m}$ wires at $80 \mu \mathrm{m}$ spacing. The micromesh is held above the anode planes with a uniform gap of $128 \mu \mathrm{m}$ by photo-etched standoffs. The micromesh is connected to a high voltage feed by a copper trace on the PCB. The drift gap region of $1.275 \mathrm{~cm}$ thickness is defined by a ring fabricated from polyether ether ketone (PEEK) insulator material that also has provisions for the gas inlet and outlet and gas seal orings. The drift cathode is another G10 PCB. The chamber uses a $70 \%$ argon, $30 \% \mathrm{CO}_{2}$ gas mixture, selected to give lower gain but higher current capability. The Micromegas assembly contains an ${ }^{55} \mathrm{Fe}$ calibration source embedded in the drift region.

Typical operating potentials are $-710 \mathrm{~V}$ on the drift electrode and between -400 and $-600 \mathrm{~V}$ on the micromesh, depending on the gain desired. The choice of negative polarity for the mesh was guided by Poisson Superfish [19] electromagnetic field simulations of the chamber geometry, as shown in Figure 2. In the case of the mesh held at negative potential relative to the collecting electrode, a high field region is created in the relatively narrow amplification gap between the mesh and the anode where gas gain occurs. The shape of the field near the mesh is such that it tends to focus electrons between the wires of the mesh as they move from the drift gap into the amplification region. Since the amplification occurs in the small gap region, positive ions can be cleared out more quickly. Alternatively, the configuration with the mesh held positive relative to the electrodes is more like a multiwire proportional counter, with the electrons being collected at the mesh. Ionization gain occurs near the mesh wires where the field strength is very high, proportional to $\log \left(r_{\text {wire }} / r\right)$. Some gain is also realized in the amplification gap for primary ionization produced there; however, primary electrons from the drift region do not generally cross the mesh plane and the number of primary ions produced is small. A drawback of this configuration at high current is that slowly drifting positive ions produced near the mesh wires tend to build up in the low field regions that can be seen around the mesh wires in Figure 2. A buildup of positive ions near the mesh wires has the effect of increasing the effective wire diameter, $r_{\text {wire }}$, and so this type of chamber generally has less gain as beam current increases. We found by measurements in proton beams of the gain using the two polarities that the mesh held negative gives the ability to produce higher gains and that the gain is more stable with respect to variations in the beam current. There are also low field regions in the configuration with the mesh negative, but they tend to be located just above the mesh wires in the drift gap, where an accumulation of positive space charge may tend to defocus electrons drifting into the amplification region; however, the gain of the device is observed to be more stable.

A preamplifier board was designed for the readout of ionization current on each of the five channels (Figures 3 and 4). Each preamplifier board has a total of ten channels. The power module derives stabilized $\pm 5 \mathrm{~V}$ from an external power supply and an additional $-1 \mathrm{~V}$ reference voltage to which the current through the input stage is compared. The current is fed into an operational amplifier and each channel supports two different gain settings individually selectable via an external digital I/O bit to achieve a wider dynamic range. The relative high/low gain settings were selected to accommodate the different beam intensity produced by double-scattered and modulated-scanned delivery methods. The net gain of the overall system can be changed in several ways: there is a factor of 5 available in the digitizer gain, a factor of 40 in the high/low amplifier setting, and a factor of 200 in the gas gain for the mesh voltage range that we have considered. Additional adjustments can be accomplished with different gas choices which we will explore in a future study.

The analog outputs from the amplifiers are connected to a DATAQ DI-720 which was configured to sample each channel at $1 \mathrm{kHz}$. The signals are digitized, serialized, and transferred to a PC connected to the DATAQ by ethernet. Each channel is read at a $1 \mathrm{~ms}$ sampling interval for the experiments described here, though faster rates are possible with the DI-720.

The chamber assembly was irradiated with beams of protons at the Roberts Proton Therapy Center at the University of Pennsylvania. The cyclotron and beamline were designed by and are operated by ion beam applications (IBAs). The facility was designed to deliver therapeutic beams of up to $230 \mathrm{MeV}$ protons to five treatment rooms using three different delivery techniques. The first is a double-scattered beam and is the least intense in terms of instantaneous dose (ionization) rate. Beamline components spread the beam to the maximum treatment field size $(\sim 20 \mathrm{~cm})$ and then collimate to a target-specific treatment area using a varian 


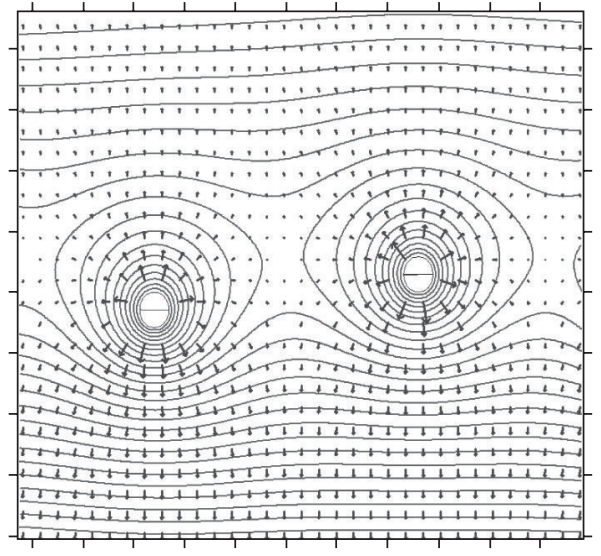

(a)

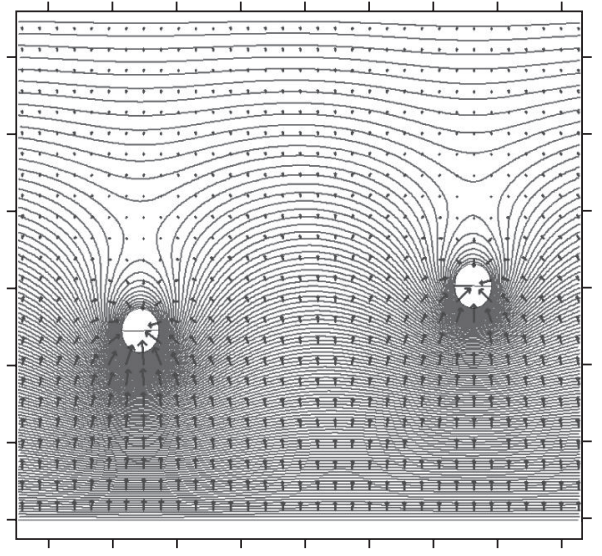

(b)

FIgURE 2: Electromagnetic field simulations of the Micromegas geometry using Poisson Superfish with the micromesh held positive (a) or negative (b) relative to the electrode at the bottom of the plots.

Proton monitor preamp board layout

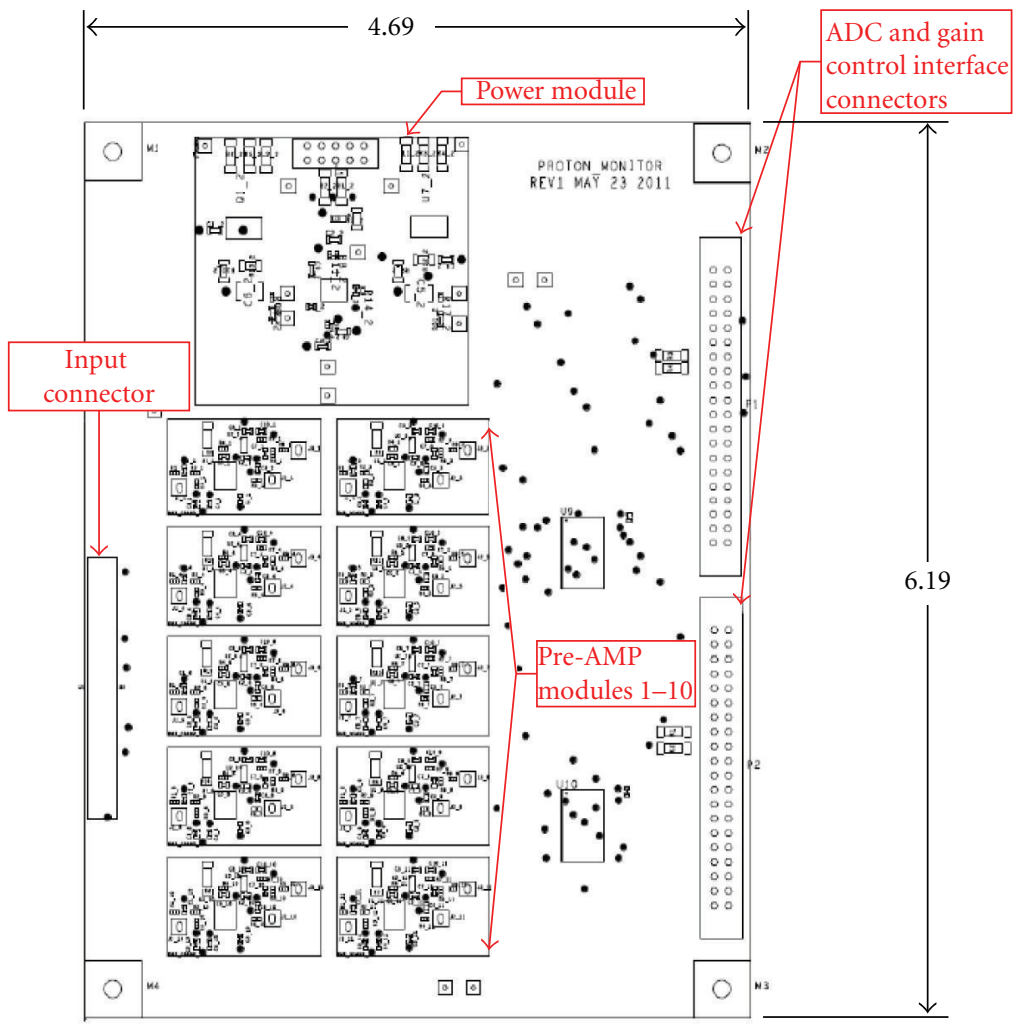

FIGURE 3: Schematic showing the layout of the preamplifier board. The power module (top) is supplied with $\pm 6 \mathrm{~V}$ by ribbon cable and provides power to the 10 amplifier modules. Ribbon connectors are used to connect the chamber channels (left) and the ADC data acquisition module (right). The preamp dimensions are indicated in inches.

multileaf collimator (MLC) comprised of 100 motorized tungsten leaves. The second type of beam is the uniformscanned beam, which is also collimated with the MLC, but the beam is magnetically scanned behind the collimator to generate larger field sizes. This delivery technique produces higher instantaneous dose rates at the time the beam is swept across the measurement volume. Finally, modulatedscanned beams, which are magnetically scanned only and not collimated, are finely focused with additional quadrupole magnets and are the most intense. We present here results obtained using the double-scattered and uniform-scanning delivery techniques. Experiments with modulated-scanned 


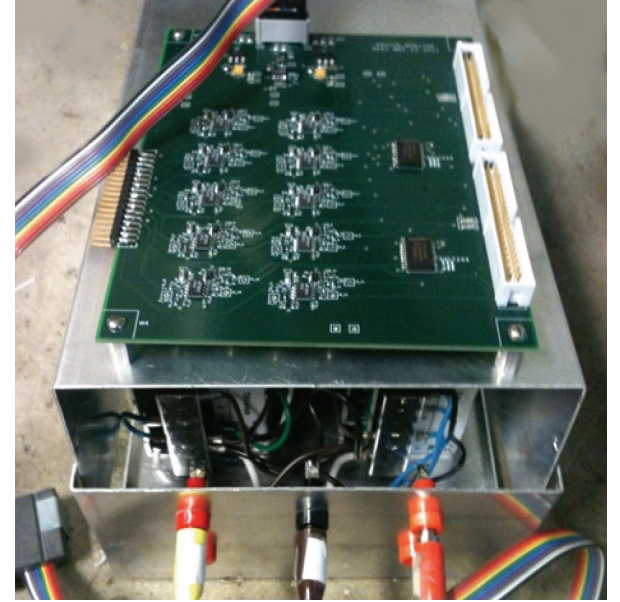

Figure 4: Photograph of the preamplifier module. Power is supplied by the ribbon cable shown. The chamber channels are connected at left and the data acquisition module interfaces with the ribbon connectors at right.

beams have also been successful but are ongoing and will be reported later. The beam range, defined as the waterequivalent depth at which the dose is $90 \%$ of the peak, that was used for these experiments was $17.5 \mathrm{~cm}$, corresponding to a mean kinetic proton energy of about $170 \mathrm{MeV}$. The beam current at cyclotron extraction was $3 \mathrm{nA}$. The transport efficiency to the treatment rooms is nominally of order $10 \%$ at this beam energy. For some experiments we have delivered spread-out Bragg peaks (SOBPs) which are modulated using a range modulator wheel. We used SOBPs of $10 \mathrm{~cm}$ modulation (i.e., the length of the flat, high-dose part of the SOBP in the depth direction). In other experiments, a single Bragg peak was delivered by stopping the modulator wheel on a particular segment. We used the MLC to collimate to small fields by opening a single leaf pair. The effective leaf width is $4.5 \mathrm{~mm}$ and the leaf pair was opened between 1 and $5 \mathrm{~mm}$ as indicated for the experiment.

The entire chamber assembly was placed in a Faraday cage of 1/16 inch copper sheet for noise shielding and the Faraday cage was placed on top of the treatment couch near the isocenter position for the treatment room. An additional ionization chamber, composed of two sheets of copper clad G10, was located on top of the Faraday cage, upstream of the Micromegas chamber. The data from that ionization chamber was used for other experiments and is not discussed here. For the spread out and single Bragg peak measurements, an acrylic tank of dimensions $12 \mathrm{~cm} \times$ $12 \mathrm{~cm}$ transverse $\times 30 \mathrm{~cm}$ depth was placed on top of the ionization chamber and filled with water. A drain pipe was used to siphon water from the tank at a controlled rate and data was collected continuously with the proton beam on while water flowed out of the tank. The total water-equivalent depth of the acrylic tank bottom, the ionization chamber, the top copper sheet of the Faraday cage, and the cathode G10 board of the Micromegas chamber is calculated to be $2.2 \mathrm{~cm}$ using stopping power ratios to water from the NIST PSTAR database $[20,21]$ for $170 \mathrm{MeV}$ protons.

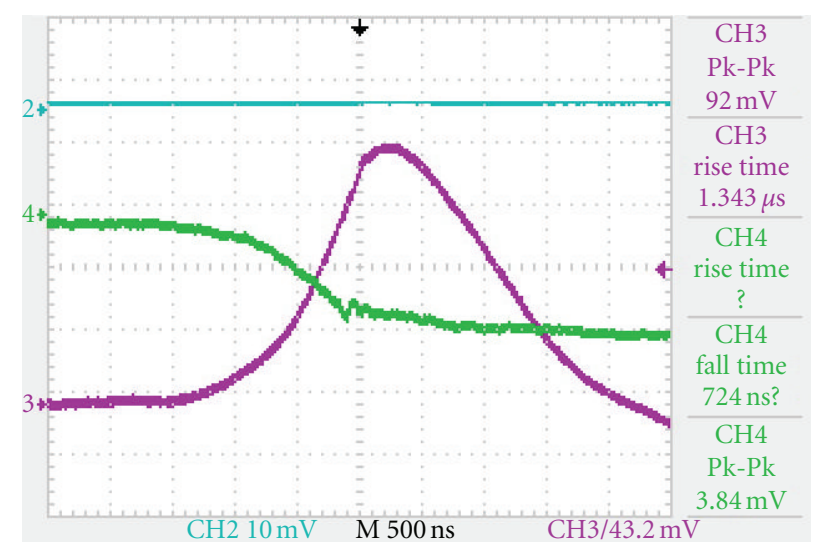

Figure 5: Average of current pulses from a ${ }^{55} \mathrm{Fe}$ test source (green trace) and the calibration signal through a shaping amplifier (purple trace).

We measured beam transverse profiles with Gafchromic EBT2 film. A calibration curve was constructed for this batch of film by delivering uniform dose cubes of varying total number of MUs with the film located in the center of the delivered dose. The film was scanned and digitized with an Epson 10000XL flatbed scanner. The red color channel was used for the optical density measurement.

We compare some of the Micromegas chamber measurements to Monte Carlo results generated using simulation code developed at the University of Pennsylvania for the IBA proton therapy nozzles and Varian MLC using the Geant4 version 9.4 (Patch-01) toolkit [22]. Our physics list was originally provided by Jarlskog and Paganetti [23], though we had to modify it for the newer version of Geant4 by replacing G4MultipleScattering with G4eMultipleScattering for electrons and positrons and with G4hMultipleScattering for hadrons. We have implemented the modulator wheels based on IBA specification and optimized the beam current modulation using least-squares fitting in order to reproduce commissioning measurements. Our simulations reproduce the beam range to within $2 \mathrm{~mm}$ and $1 \%$ dose accuracy everywhere for both the double-scattering and uniformscanning modalities.

\section{Results}

3.1. ${ }^{55} \mathrm{Fe}$ Calibration. The chamber gas and gain calibration uses a readout connected to the mesh. Typical pulses from the source are shown in Figure 5. The pulse height spectrum of the ${ }^{55} \mathrm{Fe}$ calibration (Figure 6) can be used to correct for variations in the absolute gain due to changes in gas, voltage, temperature, or pressure since it measures the absolute gain. Figure 7 shows the peak height for $-590 \mathrm{~V}$ mesh as a function of pressure and indicates that gain corrections for pressure and temperature are of the order of a few percent. The gas gain as a function of mesh voltage is given in Figure 8.

3.2. Precision and Stability of Chamber Response. For proton therapy applications, one is typically interested in the total 


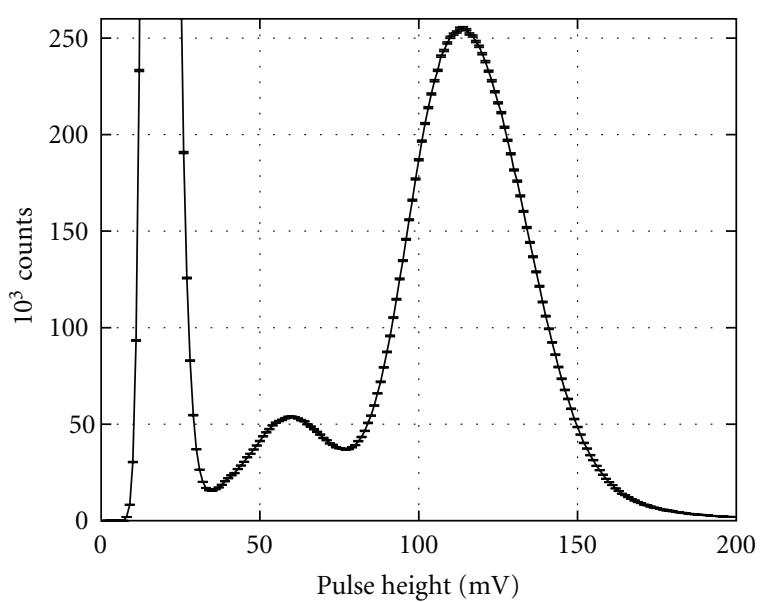

Figure 6: An example of calibration pulse height distribution from the ${ }^{55} \mathrm{Fe}$ calibration source embedded in the Micromegas chamber with mesh at $-590 \mathrm{~V}$. The pulse height is strongly dependent on the mesh potential, and somewhat dependent on the ambient temperature and pressure. The position of the main peak is used as a daily gain calibration for the Micromegas. The smaller peak is the Argon escape peak.

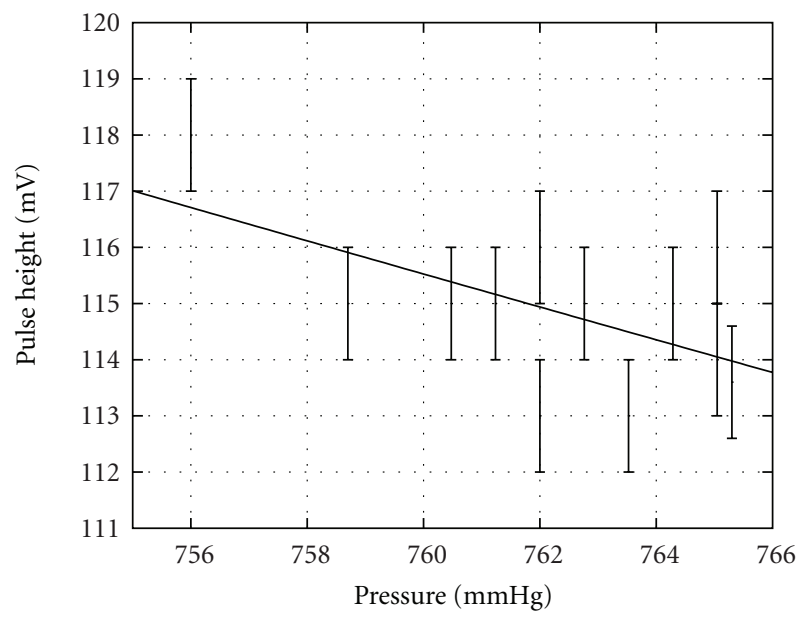

Figure 7: Pulse height versus pressure with ${ }^{55} \mathrm{Fe}$ calibration source for $-590 \mathrm{~V}$ mesh.

dose delivered to a spatial region. The integral response of the chamber should be very stable across a wide dynamic range. We measured the precision of the integrated Micromegas chamber signal by assuming a constant beam current and delivering the proton beam to the chamber for one second intervals. For each of 42 one second beam deliveries, the total charge collected by the center channel was determined by integrating the digitized signal. We plot in Figure 9 the results of those irradiations. The standard deviation from the mean for these 42 measurements is $0.8 \%$. The data show a remarkable drift in time with a timescale of about one minute. We believe that it is the beam current that is fluctuating and not the chamber response, and so consider this value to be an upper limit on the chamber precision.

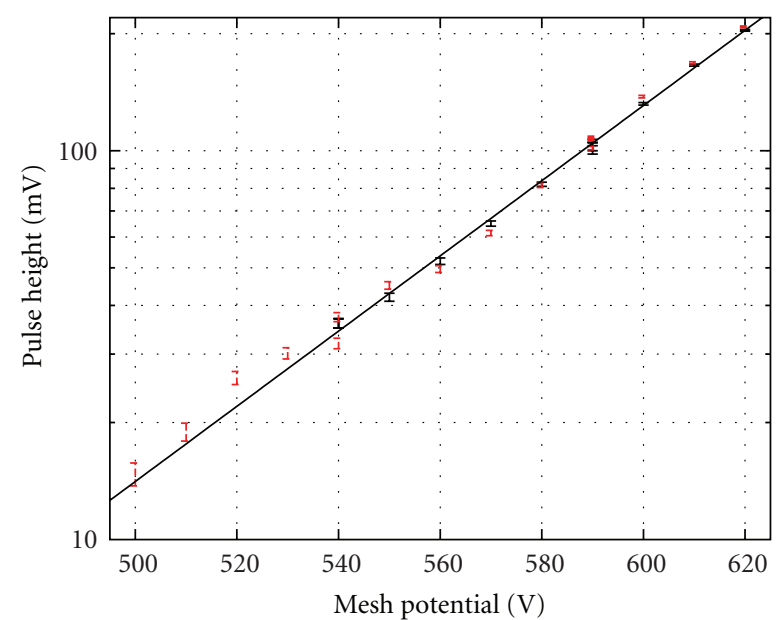

Figure 8: Gain calibration curve for the Micromegas chamber as a function of mesh potential.

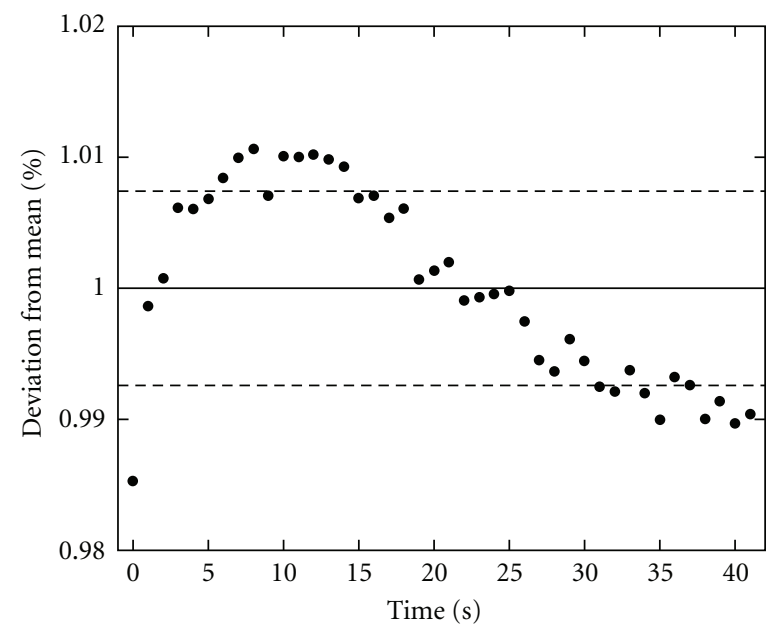

FIgURE 9: Reproducibility of the chamber response in the proton beam. The detector was irradiated in one-second intervals. The integrated response of the chamber is constant across the 42 measurements to $0.8 \%(1 \sigma)$. The signal fluctuates on about a oneminute timescale and is likely due to a drift in the beam current.

3.3. Spatial Resolution. We measured the spatial resolution of the Micromegas chamber by moving the chamber with the treatment couch across a uniform-scanned beam collimated with a $4 \mathrm{~mm} \times 4.5 \mathrm{~mm}$ aperture positioned near the boundary between channels 2 and 5. That data is shown in Figure 10. The profile of this beam was determined at the elevation of the center of the Micromegas drift gap by placing a piece of Gafchromic EBT2 film downstream of the chamber PCBs and the Faraday cage top plate. The beam is sufficiently narrow ( $4.3 \mathrm{~mm}$ FWHM) in the direction transverse to the direction of couch travel such that we collect all charge on channels 2 and 5. A ratio of the signal on channel 2 to the signal on channel 5 provides a position measurement for the beam. We measured $\sigma=1.1 \mathrm{~mm}$ for the beam position measurement using the Micromegas chamber. 


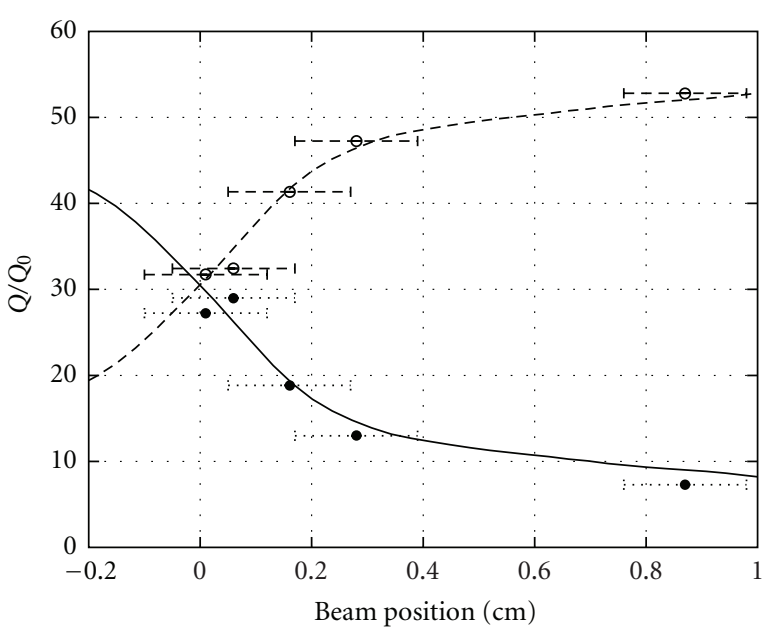

FIGURE 10: Spatial resolution measurement for the Micromegas chamber using a beam moving across the boundary between channels 2 and 5. The lines represent the fraction of the integrated beam profile that is delivered to channel 2 (dotted) and channel 5 (solid). The data points are the integrated charge $Q$ measured on the given channel relative to the charge $Q_{0}$ measured with the transmission ionization chamber in the delivery nozzle. The ratio of the signal on the two channels can be used as a beam position measurement with $\sigma=1.1 \mathrm{~mm}$.

3.4. Double-Scattered Proton Beam. The double-scattered proton beam delivery technique uses a modulator wheel with segments of varying thicknesses of Lexan and $\mathrm{Pb}$ to produce a flat spread-out Bragg peak (SOBP). The wheel rotates at a nominal $10 \mathrm{~Hz}$ and the beam is pulsed in phase with the wheel. The number of segments irradiated determines the length of the flat part of the SOBP in the depth direction. The Micromegas signal in this type of beam is shown in Figure 11. The water level was lowered continuously with the beam on a rate of $0.14 \mathrm{~mm} / \mathrm{s}$. The lower plots of Figure 11 show that the Micromegas chamber can resolve the time structure of this delivery technique. Each pulse corresponds to a rotation of the modulator wheel. Four segments of the wheel can be identified in the lower right figure, though the fourth gives a very low signal at the depth of the measurement (about $16.5 \mathrm{~cm}$ ) and is in the beam for only a few ms before the next segment. In order to produce a flat SOBP, the relative weight of Bragg peaks are set by the angular width of the corresponding segment on the modulator wheel and finetuned by modulating the beam current in phase with the wheel rotation. We show in Figure 11 that the sum of the measurements over the wheel rotation indeed produces a flat SOBP.

By smoothing individual pulses for amplifier response and then averaging over the ten pulses contained in one second of data at a particular depth, it is possible to construct profiles of the beam energy passing through the rotating wheel at different depths yielding detailed information about the wheel structure. Examples of these pulses at six different depths are shown in Figure 12.

The leading and trailing edge of the distributions can be used to extract the size of the wheel angular segments and compare them to specifications from the manufacturer. Individual wheels may vary slightly and this technique is capable of measuring the differences between wheels in different treatment rooms. The comparison for the room used in these tests is shown in Figure 13 and the agreement is excellent.

A Fourier transform of the signal collected with the double-scattering delivery is shown in Figure 14. This provides a measurement of the wheel angular frequency, which we find to be $10.0046 \pm 0.0032 \mathrm{~Hz}$.

3.5. Uniform-Scanned Proton Beam. A uniform-scanned beam of $17.5 \mathrm{~cm}$ water equivalent proton range was collimated to $5 \mathrm{~mm} \times 4.5 \mathrm{~mm}$ using the MLC. The beam is scanned magnetically at nominal $3 \mathrm{~Hz}$ in the head to foot transverse direction and $30 \mathrm{~Hz}$ in the left right direction. Figure 15 shows a typical signal in the center channel of the Micromegas chamber for this kind of delivery. Each peak arises from the beam scanning at the high frequency across the collimation hole. The first four peaks correspond to the beam moving slowly in the head-to-foot direction. The pattern is reversed as the beam is scanned back in the opposite direction. The pattern of pulses observed is consistent with the nominal frequency values, but illustrates that much more accurate measurements are possible. In particular, we get from the Fourier transform of this data (Figure 16) a measurement of the scanning frequencies: $3.1146 \mathrm{~Hz} \pm 0.2 \%$ in the slow direction, and $31.146 \mathrm{~Hz} \pm$ $0.04 \%$ in the fast direction (using the fundamental peaks). However, Figure 15 is actually 65 consecutive pulse patterns plotted with a phase shift of $0.32112 \mathrm{~s}$ between pulses. The 65 pulse trains overlay extremely well indicating that the beam scan pattern is very well controlled and reproducible. Changing the phase shift by only $0.00001 \mathrm{~s}$ disrupts the overlay of the pulses, and so this is a measurement of one of the scan frequencies which is much more precise than that from the FT: $3.1141 \mathrm{~Hz} \pm 0.002 \%$. At this level of precision one has to consider the accuracy of the data acquisition clock itself.

3.6. Bragg Peak Measurement. We delivered a pristine (unmodulated) Bragg peak to the detector by stopping the modulator wheel on the first (thinnest) segment. The beam was collimated with a $5 \mathrm{~mm} \times 4.5 \mathrm{~mm}$ aperture. The beam was not scanned. The water level was lowered continuously with the beam on at a rate of $0.14 \mathrm{~mm} / \mathrm{s}$. The data collected with the Micromegas is compared with Geant 4 simulations in Figure 17.

The measured detector signals indicate that the beam in this configuration is synched to $60 \mathrm{~Hz}$ and is on for 10.00 microseconds and off for the following 6.67 microseconds. Averaging samples over as few as 10 pulses ( 1 second) can be used to detect pulse-to-pulse variations in delivered current to high accuracy as shown in Figure 12 which shows the result of averaging 5 pulses to the left and 5 to the right and the effect of smoothing the distribution. The result is a fraction of a percent accuracy every second. 


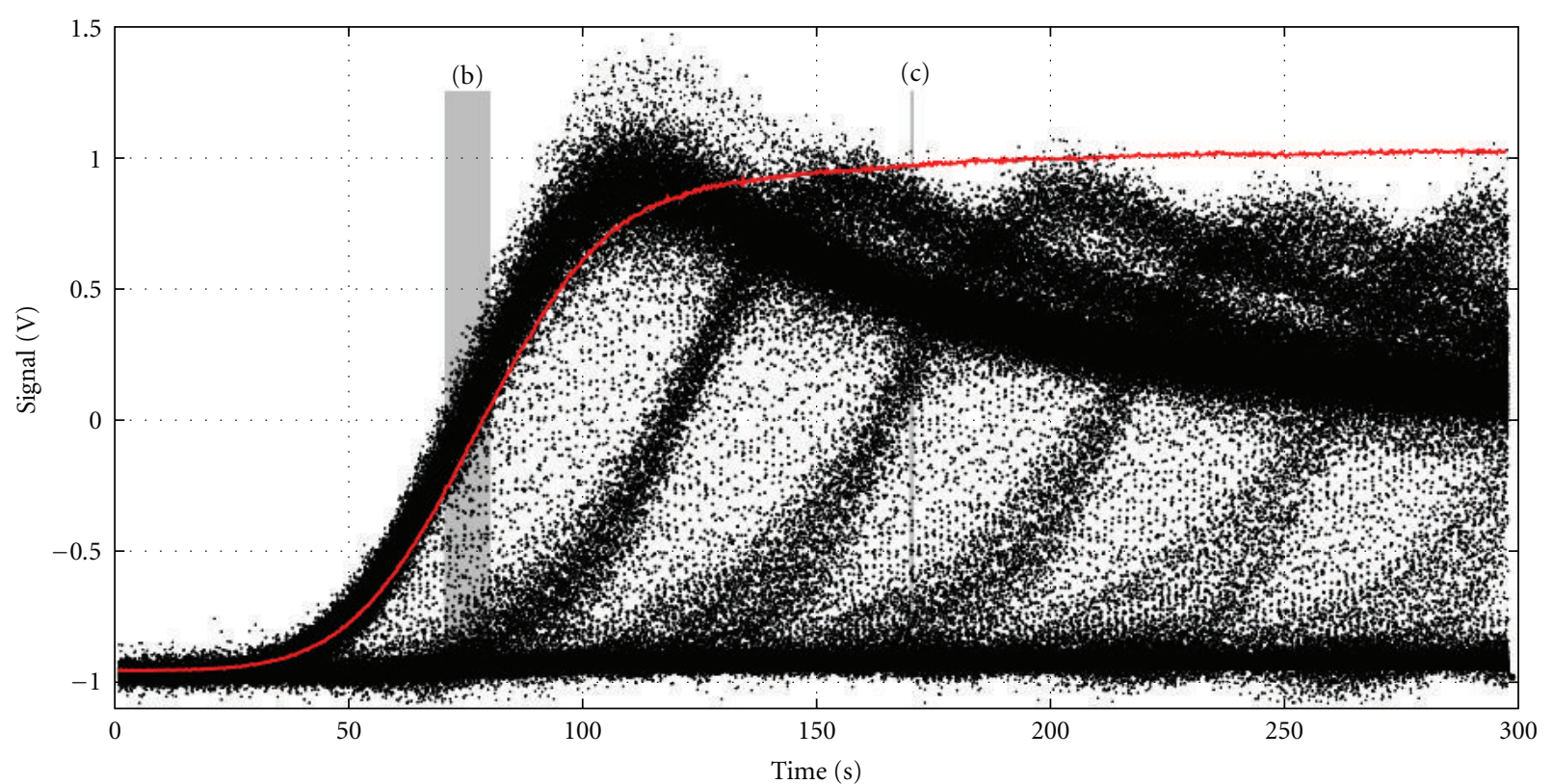

(a)

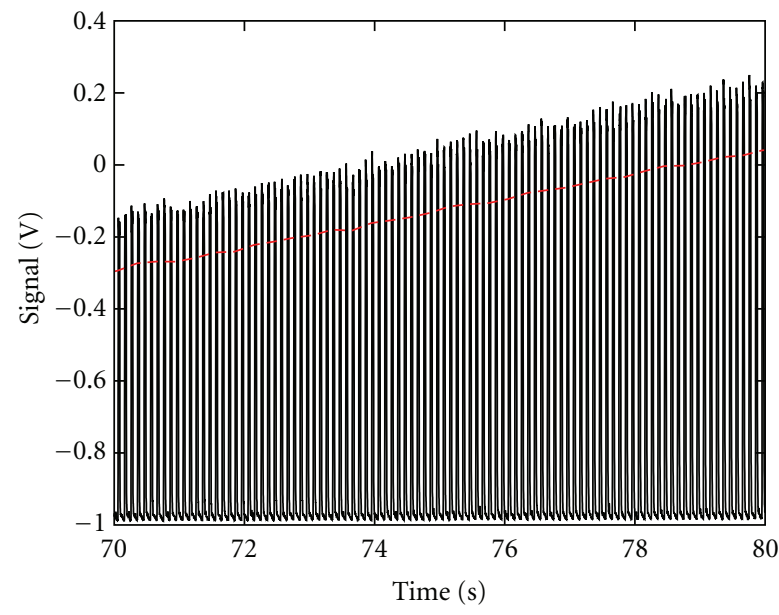

(b)

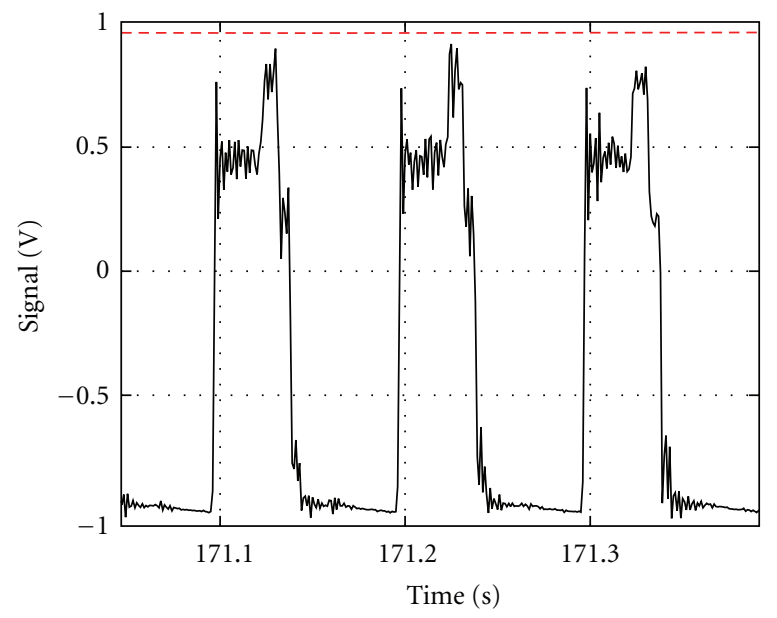

(c)

FIGURE 11: Micromegas measurement (black points, upper chart) of a proton spread-out Bragg peak (SOBP) delivered using the doublescattered technique. Five individual Bragg peaks can be seen corresponding to the first (most distal) five segments on the modulator wheel. The five Bragg peaks combine to produce a flat SOBP, as shown by the red line, which was obtained by summing the Micromegas signal over three complete wheel rotations. Two regions are highlighted for closer examination: ((b) bottom left) showing the series of pulses generated by the modulator wheel increasing in amplitude as the water depth lowers, effectively moving the detector into the distal falloff region, and ((c) bottom right) showing three wheel rotations at a depth where four Bragg peaks can be discerned both in the datapoints in the top chart and in the pulses in the bottom right.

\section{Discussion}

There are over two orders of magnitude difference in the instantaneous ionization rates encountered in proton therapy, from the relatively low rate double-scattered delivery to the high-rate modulated-scanning delivery, with uniform-scanning intermediate between the other two. The Micromegas chamber described in this paper is designed to resolve typical treatment dose rates for all modalities and is capable of a very wide dynamic range. The digitization gain is adjustable, each channel of the preamplifier board has two gain settings switchable by external digital control and further fine tuning of the gain is accomplished by adjusting the mesh potential using a calibration curve like that shown in Figure 8 and remeasuring the gain with the calibration channel.

The measurements indicate the Micromegas response is reproducible to better than $0.8 \%$ in a double-scattered proton therapy beam. The prospects for Micromegas for relative and absolute dosimetry appear promising. Indeed, 


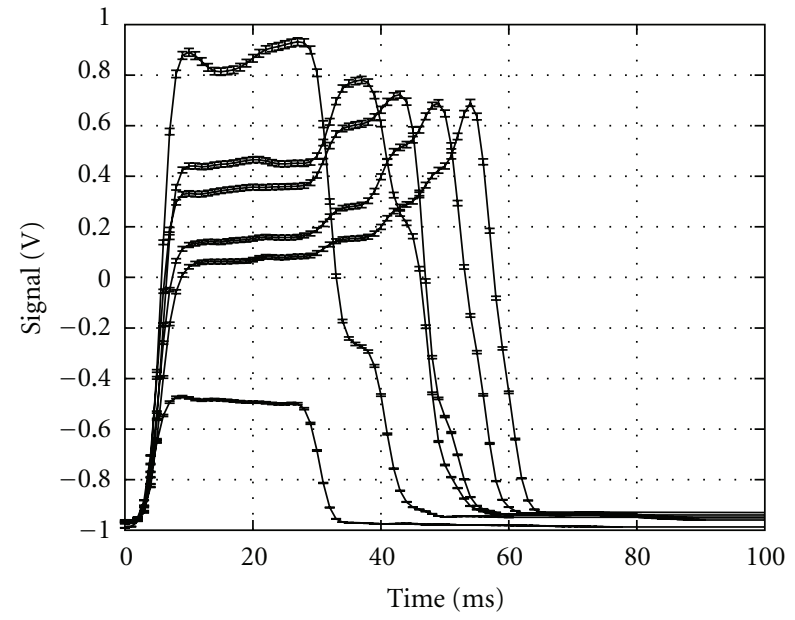

FIGURe 12: Pulses produced by the range modulator wheel at six different depths in water averaged over one second intervals. In order of increasing width, the pulses were taken from the data in Figure 11 at time $0,51,130,190$, and 237 s, corresponding to waterequivalent depths of $17.9,17.2,16.2,15.4$, and $14.8 \mathrm{~cm}$, respectively.

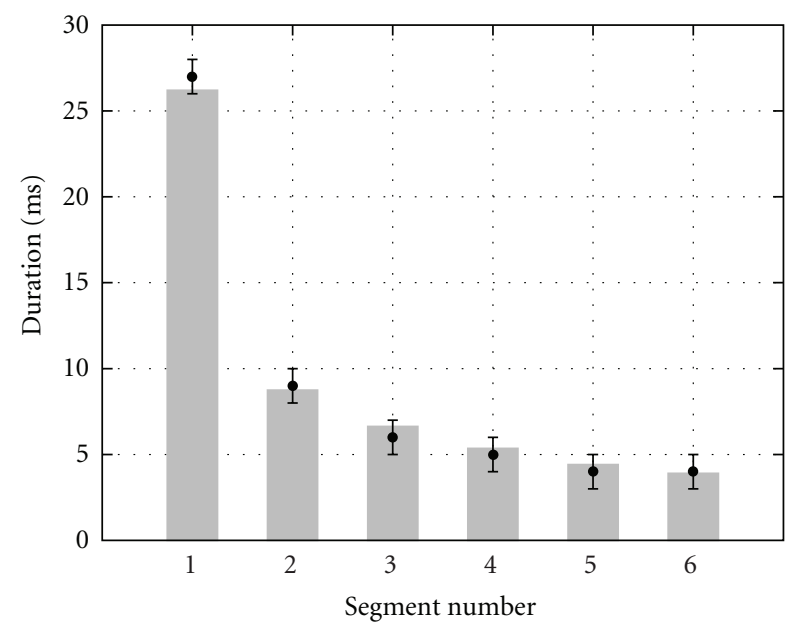

FIGURE 13: Duration (in ms) during which segments of a modulator wheel cross the beam path measured with the Micromegas (points with error bars) compared to the manufacturer data (shaded columns). The measurements were extracted from the pulses at six different depths in water averaged over one second shown in Figure 12.

while ionization chambers are currently the standard for absolute dosimetry in the field of external beam therapy, the technique used here might achieve the same absolute level of accuracy with vastly improved spatial and time resolutions. The challenge for Micromegas will be to achieve stable gain, including a precise method to correct for ambient conditions. This is commonly achieved by a feedback voltage of order less than 1 volt applied to the preamp voltage reference and calibrated using the calibration source. We continue to optimize the chamber geometry, gas composition, and operation settings and the preamplifier design and look

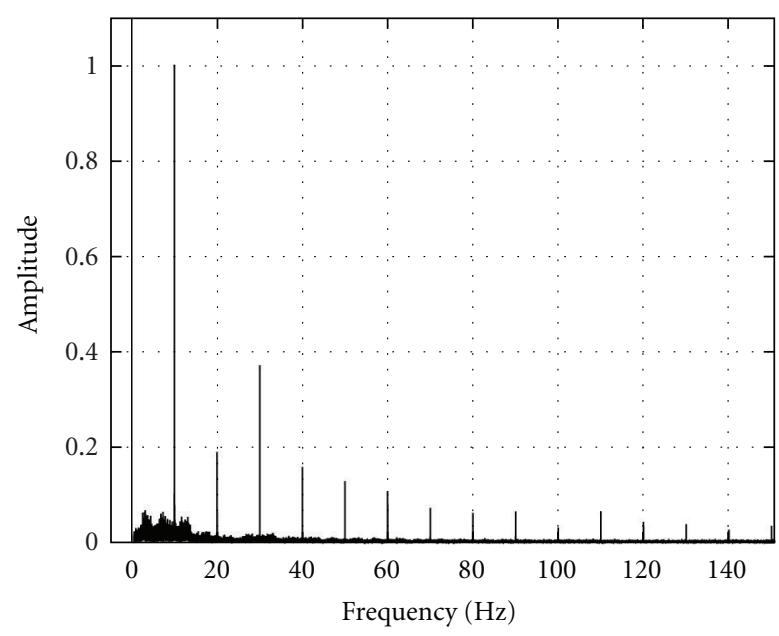

FIGURE 14: Fourier transform of ionization current collected using the double-scattered proton delivery technique. We measure the wheel rotational frequency to be $10.0046 \pm 0.0032 \mathrm{~Hz}$.

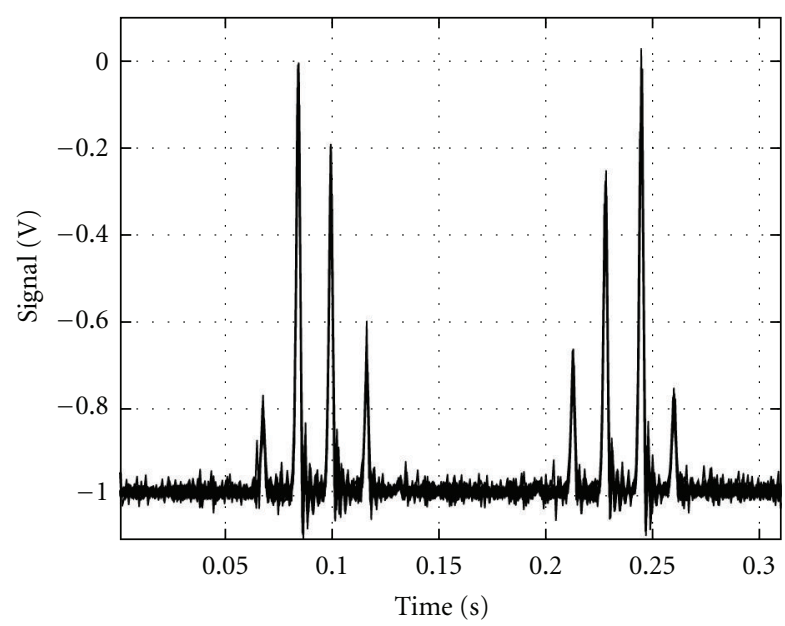

FIGURE 15: Ionization signal from the Micromegas chamber using the uniform-scanned proton delivery technique in which a relatively large Gaussian spot is magnetically scanned to produce a large, uniform fluence behind a collimating aperture. The aperture was $5 \mathrm{~mm} \times 4.5 \mathrm{~mm}$. The beam was on for 21 seconds and 65 pairs of pulse sets were collected in that time. All 65 sets are plotted here by shifting the phase of each set by $0.32112 \mathrm{~s}$.

forward to reporting in the future on its performance across the full dynamic range of proton therapy ionization rates.

The $1 \mathrm{~mm}$ spatial resolution that we demonstrate is encouraging and is in line with what is typically accepted as a clinically meaningful resolution. The commercially available $2 \mathrm{D}$ arrays of ion chambers or diodes are typically spaced at $\sim 7 \mathrm{~mm}$. We have designed a Micromegas chamber with smaller pads and look forward to resolving modulatedscanning beams as they are steered magnetically. Current therapy-grade systems produce these beams with beam sigmas $\sim 3 \mathrm{~mm}$, though that size could be driven smaller in the future as the technology improves. 


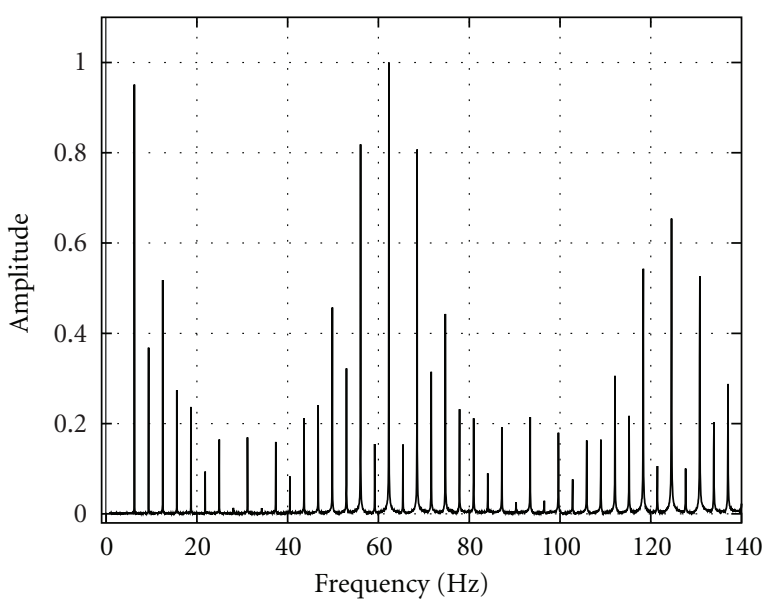

Figure 16: Fourier transform of uniform-scanning delivery data. We measured the fundamental frequencies to be 6.2291 and 62.29 Hz. The beam scanning frequencies are one-half these values, because the beam passes the collimator hole twice per scan cycle.

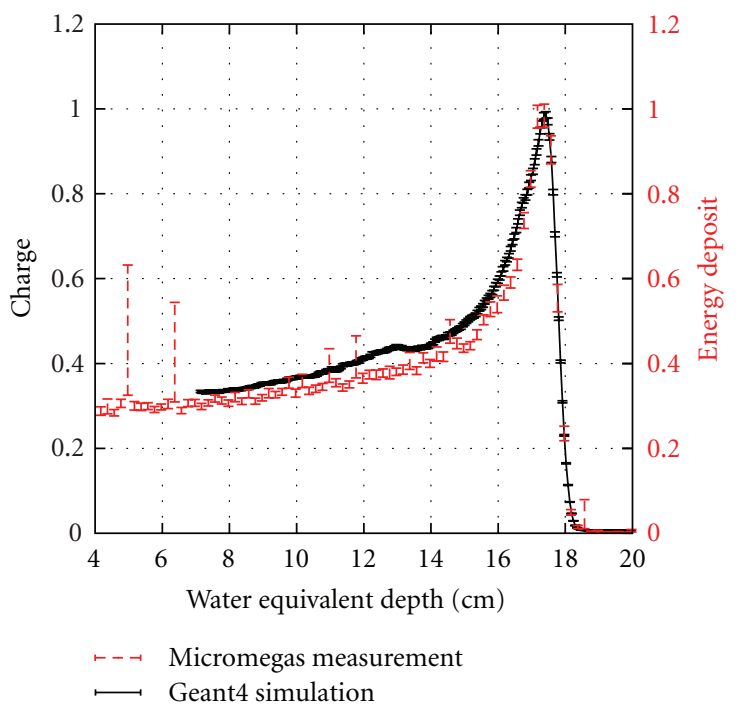

Figure 17: A proton Bragg peak measurement using the Micromegas chamber compared with Geant4 simulation of the IBA Universal Nozzle, Varian MLC, and the Micromegas detector and water column. Agreement is within 5\% across the water-equivalent depth range from $7.5-19.5 \mathrm{~cm}$. The curves are normalized at the peaks. The error bars on the Micromegas measurement represent the $0.8 \%$ dose reproducibility (see Figure 9 ) and the error bars on the simulation represent the Monte Carlo sample variance.

It is interesting that the individual segments on the modulator wheel can be identified in the data we have taken. Proton centers that are commissioning modalities that use modulator wheels may need to input the water-equivalent thicknesses of the track segments (the Varian Eclipse treatment planning system requires this information). While the proton system vendor will typically provide tables of the segment materials and thicknesses, users may want to verify the data for themselves with measurements. We are excited by the possibility of accurately and rapidly characterizing modulator wheels without resorting to tedious delivery of beam on each individual segment of the nine wheel tracks that have been commissioned at Roberts. It would also be interesting to characterize any differences between the modulator wheels across the four treatment rooms that have wheels at Roberts. Based on the specifications of the modulator wheels provided by IBA, the first four wheel segments should cross the beam path in $26,9,7$, and $5 \mathrm{~ms}$, respectively, and these values are consistent with what we have measured from Figure 11. Accurate knowledge of the shape of the beam pulses produced by the modulator wheel may be important information for a new range verification method currently under development [24].

The Fourier transform of the ionization current shown in Figure 14 indicates some noise at the rate of a few Hz. We have identified noise on the preamplifier circuit coming back from the DATAQ module. A new revision of the preamplifier board has been designed with a filter to remove this noise. We have also increased the bandwidth of the operational amplifier and improved the input compensation which should provide time resolution as low as 50 microseconds in the next version. However, we also believe that the beam current actually fluctuates at about $2 \mathrm{~Hz}$ at the few percent level. This can be seen in the treatment control rooms on the signals coming from the beam monitor ionization chambers in the nozzle. Such small fluctuations are not important for patient treatments; the system is designed to deliver a precise integral dose over millisecond or longer timescales using beam turn-off times of order a few microseconds. However in the case of rapidly scanned beams, this may no longer be negligible.

It is interesting to note that the data resolve the very stable uniform scanning pattern (Figure 15) and therefore provides a measurement of the unknown beam profile upstream of the collimator. The peak of each pulse in the Figure corresponds to a point on the (Gaussian) beam profile in the slow scanning direction, or more precisely, the beam profile convolved against the aperture window. Furthermore, the width of these pulses determines the scan velocity of the beam in the fast scan direction, given the size of the aperture in that dimension.

Other detectors tend to saturate in the high dose rate of the Bragg peak, but we have resolved the peak relative to the entrance (Figure 17). There is some disagreement between the simulations and the measurement especially around $12-13 \mathrm{~cm}$ depth. We are told by the vendor that the beam current can drift on timescales of about 10 minutes. Typically for commissioning measurements, depth dose profiles are normalized to the signal from one of the transmission ionization chambers in the nozzle to remove beam current fluctuations. In the future we will follow that method. Alternatively, the small treatment fields that we have prepared using the MLC for these experiments are much smaller than the fields used to treat patients, and this is the first data that we have collected in order to verify the Geant4 simulations for small fields. We also point out that there are features in the proton inelastic cross section data that the Geant4 physics models do not reproduce [25]. We intend to investigate this further. 


\section{Conclusions}

Micromegas is a gas-filled detector technology that can be manufactured in bulk at low cost. We have demonstrated a new use for Micromegas as a beam monitor for proton therapy, and the data presented here indicates that Micromegas holds promise as a high spatial and time resolution measurement device for proton therapy. We believe the design could be adapted to heavier ion beams. We look forward to continuing to improve the designs and will publish additional findings in the future.

\section{Acknowledgments}

The authors thank Rui De Oliveira and the CERN team for manufacturing the mesh layer for the detector used in many of these measurements and members of the University of Pennsylvania Electronics Group for design and manufacture of the electronics. The authors are grateful to Professor Chris Ainsley for sharing his Monte Carlo code for the IBA Universal Nozzle and Varian MLC and to Professor Harald Paganetti for providing the code for his Geant4 physics list [23]. They are also especially grateful to Jake Hindenburg of IBA for his knowledge of the IBA system and for helping to setup the irradiation scenarios. This work was supported by the US Army Medical Research and Materiel Command under Contract Agreement no. DAMD17-W81XWH-04-2-0022. Opinions, interpretations, conclusions, and recommendations are those of the authors and are not necessarily endorsed by the US Army.

\section{References}

[1] M. Jermann, 2012, http://ptcog.web.psi.ch/ptcentres.html.

[2] A. Smith, "Vision 20/20: proton therapy," Medical Physics, vol. 36, no. 2, p. 556, 2009.

[3] U. Schneider, J. Besserer, P. Pemler et al., "First proton radiography of an animal patient," Medical Physics, vol. 31, no. 5, pp. 1046-1051, 2004.

[4] R. W. Schulte, V. Bashkirov, T. Li et al., "Conceptual design of a proton computed tomography system for applications in proton radiation therapy," IEEE Transactions on Nuclear Science, vol. 51, no. 3, pp. 866-872, 2004.

[5] L. Johnson, B. Keeney, G. Ross et al., "Initial studies on proton computed tomography using a silicon strip detector telescope," Nuclear Instruments and Methods in Physics Research A, vol. 514, no. 1-3, pp. 215-223, 2003.

[6] S. N. Penfold, A. B. Rosenfeld, R. W. Schulte, and H.F. W. Sadrozinksi, "Geometrical optimization of a particle tracking system for proton computed tomography," Radiation Measurements, vol. 46, no. 12, pp. 2069-2072, 2011.

[7] H.-M. Lu, "A point dose method for in vivo range verification in proton therapy," Physics in Medicine and Biology, vol. 53, no. 23, pp. N415-N422, 2008.

[8] M. Mumot, C. Algranati, M. Hartmann, J. M. Schippers, E. Hug, and A. J. Lomax, "Proton range verification using a range probe: definition of concept and initial analysis," Physics in Medicine and Biology, vol. 55, no. 16, pp. 4771-4782, 2010.

[9] B. Arjomandy, N. Sahoo, X. Ding, and M. Gillin, "Use of a twodimensional ionization chamber array for proton therapy beam quality assurance," Medical Physics, vol. 35, no. 9, pp. 3889-3894, 2008.

[10] B. Hartmann, J. Telsemeyer, L. Huber, B. Ackermann, O. Jkel, and M. Martikov, "Investigations of a flat-panel detector for quality assurance measurements in ion beam therapy," Physics in Medicine and Biology, vol. 57, p. 51, 2012.

[11] S. N. Boon, P. van Luijk, T. Bohringer et al., "Performance of a fluorescent screen and CCD camera as a two-dimensional dosimetry system for dynamic treatment techniques," Medical Physics, vol. 27, no. 10, pp. 2198-2208, 2000.

[12] S. Beddar, L. Archambault, N. Sahoo et al., "Exploration of the potential of liquid scintillators for real-time 3D dosimetry of intensity modulated proton beams," Medical Physics, vol. 36, no. 5, p. 1736, 2009.

[13] M. Titov, "New developments and future perspectives of gaseous detectors," Nuclear Instruments and Methods in Physics Research A, vol. 581, pp. 25-37, 2007.

[14] Y. Giomataris, P. Rebourgeard, J. P. Robert, and G. Charpak, "MICROMEGAS: a high-granularity positionsensitive gaseous detector for high particle-flux environments," Nuclear Instruments and Methods in Physics Research A, vol. 376, no. 1, pp. 29-35, 1996.

[15] Y. Giomataris, "Development and prospects of the new gaseous detector 'Micromegas", Nuclear Instruments and Methods in Physics Research A, vol. 419, pp. 239-250, 1998.

[16] I. Giomataris, "High rate applications of Micromegas and prospects," in Proceedings of the International Symposium On Detector Development For Particle, Astroparticle And Synchrotron Radiation Experiments (SNIC '06), 2006.

[17] Y. Giomataris, R. D. Oliveira, S. Andriamonje et al., "Micromegas in a bulk," Nuclear Instruments and Methods in Physics Research A, vol. 560, pp. 405-408, 2006.

[18] R. Oliveira, V. Peskov, F. Pietropaolo, and P. Picchi, "First tests of MICROMEGAS and GEM-like detectors made of a resistive mesh," IEEE Transactions on Nuclear Science, vol. 57, no. 6, pp. 3744-3752, 2010.

[19] K. Halbach and R. F. Holsinger, "SUPERFISH-a computer program for evaluation of RF cavities with cylindrical symmetry," Particle Accelerators, vol. 7, pp. 213-222, 1976.

[20] http://physics.nist.gov/PhysRefData/Star/Text/PSTAR.html.

[21] "ICRU report 49: Stopping powers and ranges for protons and alpha particles," 1993.

[22] S. Agostinelli, J. Allison, K. Amako et al., "Geant4-a simulation toolkit," Nuclear Instruments and Methods in Physics Research A, vol. 506, pp. 250-303, 2003.

[23] C. Z. Jarlskog and H. Paganetti, "Physics settings for using the Geant 4 toolkit in proton therapy," IEEE Transactions on Nuclear Science, vol. 55, no. 3, pp. 1018-1025, 2008.

[24] B. Gottschalk, S. Tang, E. H. Bentefour, E. W. Cascio, D. Prieels, and H.-M. Lu, "Water equivalent path length measurement in proton radiotherapy using time resolved diode dosimetry," Medical Physics, vol. 38, no. 4, p. 2282, 2011.

[25] http://geant4.web.cern.ch/geant4/results/validation\%5C_ plots/cross\%5C_sections/hadronic/inelastic/test1/inelastic .shtml 

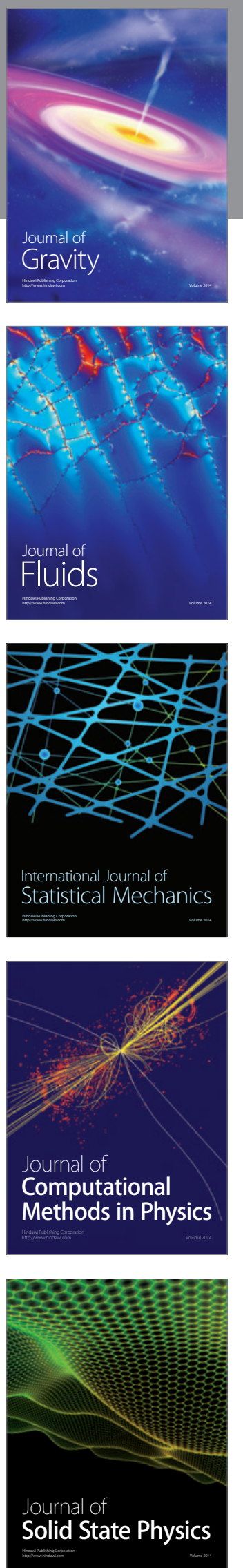

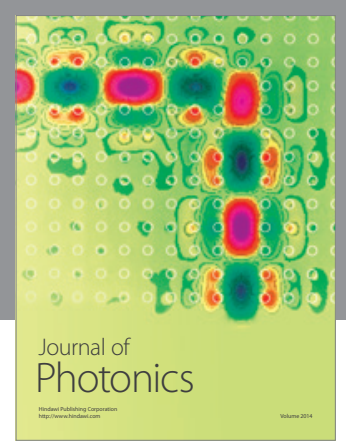

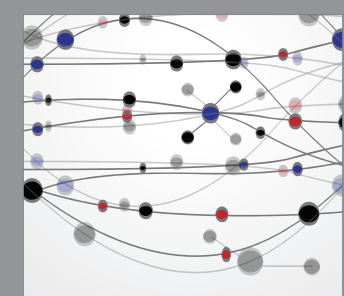

The Scientific World Journal
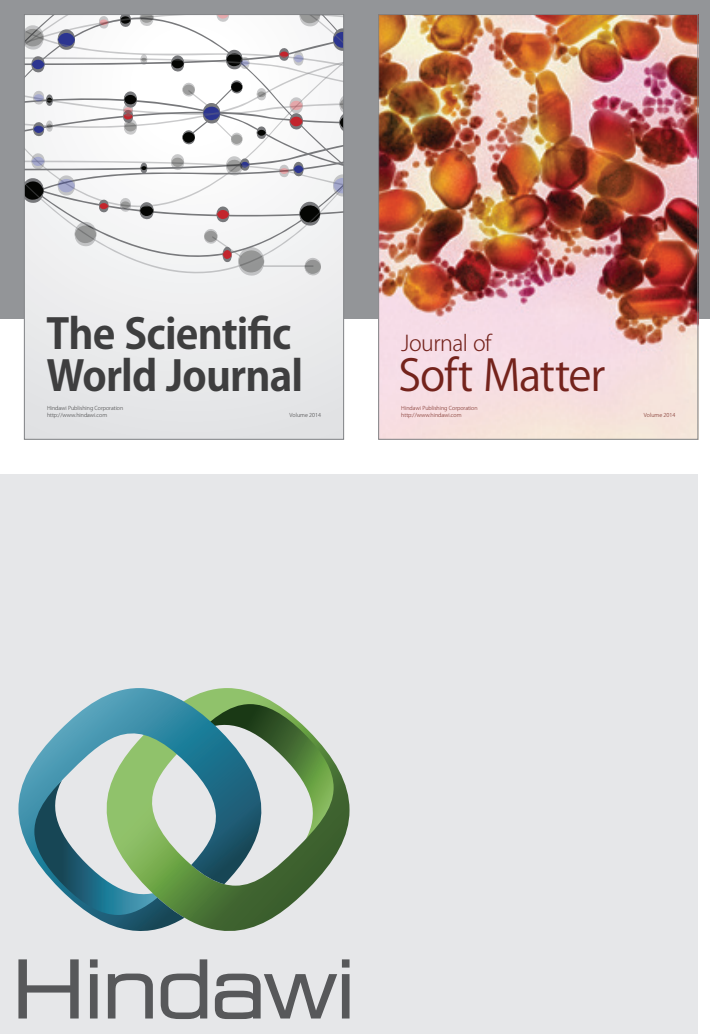

Submit your manuscripts at

http://www.hindawi.com
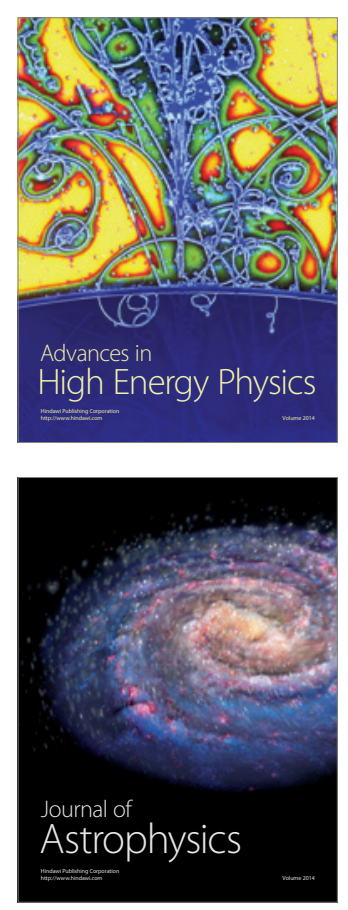
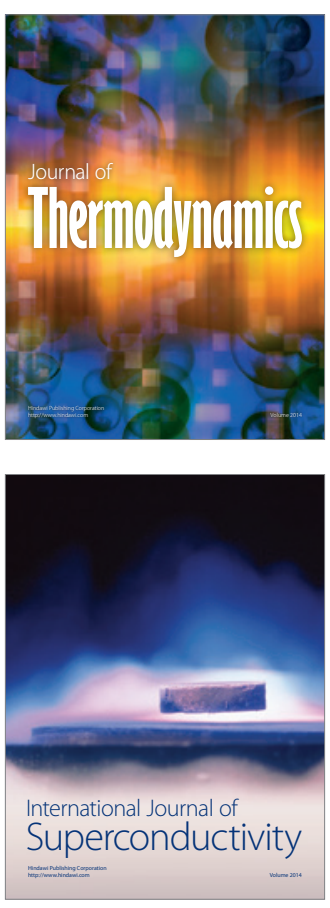
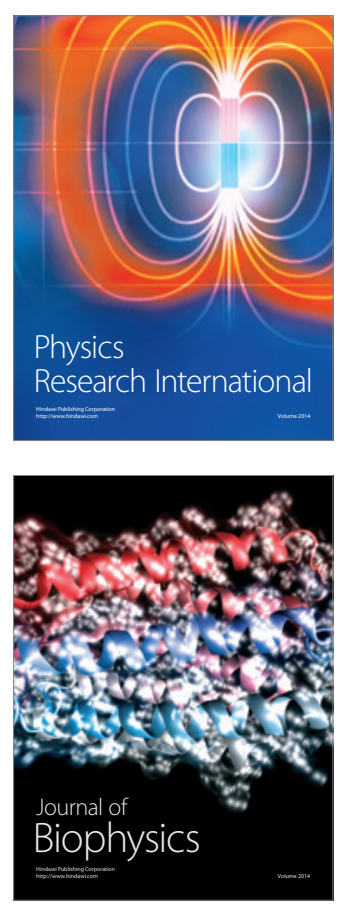
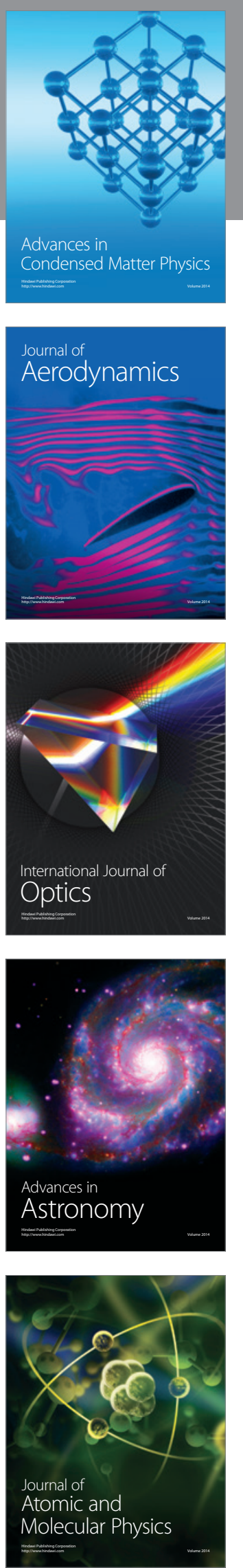